\title{
Overexpression of LRRC59 Is Associated with Poor Prognosis and Promotes Cell Proliferation and Invasion in Lung Adenocarcinoma
}

This article was published in the following Dove Press journal: OncoTargets and Therapy

\author{
Dong $\mathrm{Li} \mathbb{D}^{1, *}$ \\ Ying Xing $\mathbb{D}^{2, *}$ \\ Tiannv $\operatorname{Tian}^{3}$ \\ Yanan Guo ${ }^{3}$ \\ Jing Qian ${ }^{3,4}$
}

'Department of Thoracic Surgery, Huzhou Central Hospital, Huzhou, Zhejiang, People's Republic of China;

${ }^{2}$ Department of Gastroenterology, The

72nd Army Hospital of the People's Liberation Army of China, Huzhou,

Zhejiang, People's Republic of China;

${ }^{3}$ Huzhou University Schools of Nursing and Medicine, Huzhou University, Huzhou, Zhejiang, People's Republic of China; ${ }^{4}$ Key Laboratory of Vector Biology and Pathogen Control of Zhejiang Province, Huzhou, Zhejiang, People's Republic of China

*These authors contributed equally to this work

\begin{abstract}
Aim: LRRC59 (leucine-rich repeat-containing protein 59) is a ribosome-binding protein that also interacts with fibroblast growth factors. Limited investigations revealed a possible role of LRRC59 in the aggressive phenotype of breast cancer. However, whether LRRC59 contributes to the progression of lung cancer remains unclear.

Materials and Methods: In this study, an online TCGA-based survival analysis software (GEPIA2) was used to estimate the prognostic value of LRRC59 mRNA expression level for lung cancer. Cell Counting Kit-8 assay, colony-forming assay, cell cycle analysis, and transwell assay were used to assess the biological functions of LRRC59 in lung cancer cells. Then, 94 lung adenocarcinoma (LUAD) patient tissues were collected to examine the expression level of LRRC59 by the tissue microarray (TMA)-based immunohistochemistry staining (IHC). Univariate Kaplan-Meier and multivariate Cox regression analyses were performed to evaluate the prognostic value of LRRC59 protein expression in LUAD.

Results: Higher mRNA level of LRRC59 was significantly associated with worse survival for lung adenocarcinoma, but not for lung squamous cell carcinoma. Knockdown of LRRC59 by shRNA apparently inhibited cell proliferation and colony formation in both H1299 and A549 cells. The G1/S phase arrest induced by LRRC59 depletion was observed in A549 and H1299 cells. Besides, the silencing of LRRC59 decreased cell migrative and invasive abilities. Moreover, TMA-based IHC showed that LRRC59 was highly expressed in LUAD tissues and closely associated with lymph node metastasis $(\mathrm{P}<0.001)$, TNM stage $(\mathrm{P}<0.001)$, and histological differentiation $(\mathrm{P}=0.007)$. Further multivariate analysis suggested that LRRC59 overexpression was an independent prognostic factor in LUAD.
\end{abstract}

Conclusion: LRRC59 may serve as a novel biomarkers and therapeutic target for LUAD clinical practice.

Keywords: LRRC59, lung adenocarcinoma, proliferation, metastasis, prognosis

\section{Introduction}

Lung cancer ranks among the most frequent cause of cancer-related deaths with an approximate 5-year overall survival rate of less than $18 \%$. The key reason for poor lung cancer treatment is the inadequate potential biomarkers for predicting and diagnosing non-small cell lung cancer (NSCLC) for molecular targeted or personalized therapies. Lung adenocarcinoma (LUAD) is currently the most common histologic type of lung cancer. ${ }^{1}$ Therefore, exploring the underlying mechanisms and related molecules during lung adenocarcinoma development and progression seems of great importance.
Correspondence: Jing Qian Huzhou University Schools of Nursing and Medicine, Huzhou University, Huzhou, Zhejiang Province 313000,

People's Republic of China

$\mathrm{Tel}+8613905723707$

Email 02300@zjhu.edu.cn 
LRRC59 was first described as a ribosome receptor on the ER that also interacts with fibroblast growth factors $(\mathrm{FGF} 1),{ }^{2-4}$ it is a tail-anchored ER membrane protein containing a small $\mathrm{C}$-terminal domain facing the $\mathrm{ER}$ lumen, a leucine-rich repeat (LRR) domain, and a putative coiled-coil domain facing the cytosol. Zhen et al showed that LRRC59 was explicitly required for the nuclear import of FGF1. ${ }^{5}$ Also, Pallai et al found that LRRC59 was a novel binding partner of the oncoprotein cancerous Inhibitor of PP2A (CIP2A) and was required for CIP2A nuclear import. ${ }^{6}$ Recently, Yu et al showed that a fusion transcript of LRRC59-FLJ60017 to be present in prostate carcinomas, ovarian cancer, esophageal adenocarcinoma, non-small cell lung cancer and glioblastoma multiforme. $^{7}$ Meanwhile, they founded that detection of the fusion transcripts along with high Gleason scores was predictive of prostate carcinoma recurrence. Furthermore, Terp et al demonstrated that the altered expression of LRRC59 was correlated with the metastatic capability of breast cancer cell lines. ${ }^{8}$ Although the above information suggests that LRRC59 might be related to cancer, the biological functions of LRRC59 are yet to be identified directly in lung cancer.

In the present study, we aimed to investigate the expression pattern and clinical significance of LRRC59 in lung cancer and its critical role in lung cancer cell proliferation and metastasis by silencing in vitro. Our results suggested that LRRC59 is an oncogenic factor that contributes to cancer proliferation and metastasis. LRRC59 may be a novel biomarkers and therapeutic target in lung cancer clinical practice.

\section{Materials and Methods}

\section{Online Survival Analysis}

Differential expression of LRRC59 in lung cancer and normal tissues was analyzed using the online Gene Expression Profiling and Interactive Analysis (GEPIA2; http://gepia2.cancer-pku.cn/\#index) database. GEPIA2 is a newly developed interactive web server for the analysis of RNA sequencing data derived from 9736 tumors and 8,587 healthy samples from The Cancer Genome Atlas (TCGA) and Genotype-Tissue Expression datasets. ${ }^{9}$ GEPIA provides customizable functions including tumor/ normal differential expression analysis, profile plotting, correlation analysis, patient survival analysis, detection of similar genes, and dimensionality reduction analysis. The $\left|\log _{2} \mathrm{FC}\right|$ cutoff of the expression of proposed biomarker was 1 . The p-value cutoff of the expression of proposed biomarker was 0.01 . All boxplot analysis used $\log _{2}($ TPM +1$)$ for log-scale.

The GEPIA2 survival analysis tool was used to assess the relationship between LRRC59 mRNA expression and lung cancer prognosis based on gene expression levels and uses a Log rank test for hypothesis evaluation. We selected a hazards ratio (HR) based on the Cox PH model and also the $95 \%$ confidence interval information to show the $95 \%$ confidence interval (CI) as the dotted line. The LRRC59 expression threshold of $50 \%$ (median value) was determined to split the LRRC59 high-expression and lowexpression cohorts. Therefore, samples with LRRC59 expression levels higher and lower than 50\% were applied as the high-expression cohort (cuto-high) and the lowexpression cohort (cuto-low), respectively.

\section{Tissue Samples and Cell Culture}

A total of 94 newly diagnosed primary LUAD patients (Aged 62 \pm 10 ) were recruited in Huzhou Centre Hospital between July 2004 to April 2009, and their clinicopathological characteristics were retrieved (Table 1). Paired tissue specimens were collected during surgery, including fresh lung tumor and matched adjacent tissues. Two pathologists independently reviewed all pathologic specimens. This study was approved by the Ethics Committee of Huzhou Central Hospital in accordance with the Declaration of Helsinki (approval No.20181102-01), and all the participants provided informed consent. The A549 and H1299 cell lines were obtained from American Type Culture Collection though Shanghai Outdo Biotech Company in china. A549 cells was cultured in Dulbecco's modified Eagle's medium (DMEM, Gibco, Shanghai, China), while H1299 cells was cultured in RPMI-1640 medium (Gibco), containing 10\% fetal bovine serum and $1 \%$ penicillin/streptomycin, and incubated in a humidified $5 \% \mathrm{CO}_{2}$ atmosphere at $37{ }^{\circ} \mathrm{C}$.

\section{Immunohistochemical Analysis (IHC)}

The tissue microarray (TMA) was generated and consisted of 94 LUAD tissues. All stainings were scored based on the staining intensity and extensity of positive cells, the intensity $(0=$ genitive, $1=$ weak, $2=$ moderate, and $3=$ strong) and extensity ( $0=9 \%$ or less of cells stained positive; $1=10 \%-25 \% ; 2=26 \%$ to $50 \% ; 3=51 \%$ to $80 \%$; and $4=81 \%$ or more) of tumor staining were evaluated. The positive cell density of each core was counted by two independent investigators blind to clinical outcome and knowledge of the clinicopathological data. The final 
Table I Association Between LRRC59 Expression and Clinicopathologic of LUAD Patients

\begin{tabular}{|c|c|c|c|c|}
\hline \multirow[t]{2}{*}{ Variables } & \multirow[t]{2}{*}{ Case } & \multicolumn{2}{|c|}{$\begin{array}{l}\text { LRRC59 Protein } \\
\text { Expression Level }\end{array}$} & \multirow[t]{2}{*}{$P$-value } \\
\hline & & $\begin{array}{l}\text { Low } \\
(\mathrm{N}=38)\end{array}$ & $\begin{array}{l}\text { High } \\
(\mathbf{N}=56)\end{array}$ & \\
\hline $\begin{array}{l}\text { Age (Year) } \\
\quad \leq 62 \\
>62\end{array}$ & $\begin{array}{l}49 \\
45\end{array}$ & $\begin{array}{l}24 \\
14\end{array}$ & $\begin{array}{l}25 \\
31\end{array}$ & 0.078 \\
\hline $\begin{array}{l}\text { Gender } \\
\text { Male } \\
\text { Female }\end{array}$ & $\begin{array}{l}51 \\
43\end{array}$ & $\begin{array}{l}19 \\
19\end{array}$ & $\begin{array}{l}32 \\
24\end{array}$ & 0.495 \\
\hline $\begin{array}{l}\text { Tumor size }(\mathrm{cm}) \\
\quad \leq 4 \\
>4\end{array}$ & $\begin{array}{l}41 \\
53\end{array}$ & $\begin{array}{l}20 \\
18\end{array}$ & $\begin{array}{l}21 \\
35\end{array}$ & 0.147 \\
\hline $\begin{array}{l}\text { Differentiation } \\
\text { Well } \\
\text { Moderate } \\
\text { Poor }\end{array}$ & $\begin{array}{l}5 \\
68 \\
21\end{array}$ & $\begin{array}{l}5 \\
28 \\
5\end{array}$ & $\begin{array}{l}0 \\
40 \\
16\end{array}$ & $0.007^{\mathrm{a}}$ \\
\hline $\begin{array}{l}\text { TNM stage } \\
\text { I } \\
\text { II } \\
\text { III/IV }\end{array}$ & $\begin{array}{l}28 \\
28 \\
38\end{array}$ & $\begin{array}{l}18 \\
13 \\
7\end{array}$ & $\begin{array}{l}10 \\
15 \\
31\end{array}$ & $0.001^{\mathrm{a}}$ \\
\hline $\begin{array}{l}\text { LN metastasis } \\
\text { Negative } \\
\text { Positive }\end{array}$ & $\begin{array}{l}40 \\
54\end{array}$ & $\begin{array}{l}24 \\
14\end{array}$ & $\begin{array}{l}16 \\
40\end{array}$ & $0.00 I^{\mathrm{a}}$ \\
\hline
\end{tabular}

Notes: Statistical analyses were performed by Pearson's $\chi^{2}$ test. ${ }^{\text {a }} \mathrm{P}<0.05$ was considered significant.

Abbreviations: LUAD, lung adenocarcinoma; TNM, T: primary tumor; N: regional lymph nodes; M: distant metastasis. LN, lymph nodes.

score was calculated by adding the strongest intensity score and the total extensity score (maximum value of 7). The cut-off value of LRRC59 expression was determined by median IHC score. We defined a sample as high LRRC59 expression when the sample had a combined score greater than 4 , and a sample was low expression LRRC59 if the combined score $\leq 4$.

\section{Quantitative Real-Time Polymerase Chain Reaction}

mRNA transcripts were quantified by quantitative realtime polymerase chain reaction (RT-qPCR). Briefly, RNAs from lung cancer cell lines were isolated with RNAiso Trizol (Invitrogen, Shanghai, China). cDNA was reversed transcribed using the PrimeScript ${ }^{\mathrm{TM}}$ RT reagent kit (Takara, Shiga, Japan). Then, cDNA templates were amplified by real-time RT-PCR using the SYBR Green
PCR Kit (Takara) by the ABI7500 System (Applied Biosystems, USA). $\beta$-actin was used as an internal control. The relative expression levels of mRNA were calculated by the $2^{-\Delta \Delta \mathrm{Ct}}$ method. The primer $(10 \mu \mathrm{M}$ for each gene) sequences were as follows: LRRC59, 5'-TGACTACTC TACCGTCGGATTT-3'(for), 5'-TTCAGGTCCAACCACT TCAGG-3'(rev); $\beta$-actin, 5'-ACGCCAACACAGTGCTGT CTG-3'(for), 5'-GGCCGGACTCGTCATACTCC-3'(rev).

\section{Short Hairpin RNA Lentivirus Transduction}

The short hairpin RNA (shRNA) lentivirus transduction approach was used to knockdown LRRC59 gene expression. Lentivirus vector and two short hairpin RNA (shRNA) targeting LRRC59 were purchased from Genepharma Shanghai. Targeting LRRC59 sequences were listed as followed: shRNA\#1:5'-CCTGGATCTGTCTTGTAATAA-3'; shRNA\# 2:5'-GCAGTGTAAAGCAGTGTGCAAA-3'. shCon:5'-AAT ACGGCGATGTGTCAGG-3', The shRNAs were packaged into lentiviruses by cotransfecting with packaging plasmids pMD2G and pSPAX2 into 293T cells. Forty-eight hours after transfection, the supernatant was collected for infection of A549/H1299 cells. Infected cells were selected with media containing puromycin $(2 \mu \mathrm{g} / \mathrm{mL})$ for 3 days.

\section{Immunoblot Analysis}

Lung cancer cell was lysed for $30 \mathrm{~min}$ on ice in RIPA buffer (50 mM Tris pH 7.4, $150 \mathrm{mM} \mathrm{NaCl}, 1 \%$ Triton X-100, 1\% Na deoxycholate, $0.1 \%$ SDS, $1 \mathrm{mM} \mathrm{Na} \mathrm{VO}_{4}, 1 \mathrm{mM}$ PMSF, $10 \mathrm{mg} / \mathrm{mL}$ leupeptin, $10 \mathrm{mg} / \mathrm{mL}$ aprotinin) that contained $1 \mathrm{mM}$ PMSF. Whole-cell lysates containing $50 \mu \mathrm{g}$ of proteins, were separated by $10 \%$ SDS-PAGE gel electrophoresis gels, the proteins were transferred to a $0.45-\mu \mathrm{m}$ hydrophobic PVDF transfer membrane (Millipore) using a semidry transfer apparatus (Bio-Rad). The transfer ran at $17 \mathrm{~V}$ for $30 \mathrm{~min}$, and blocked for $1 \mathrm{~h}$ at room temperature in a 5\% BSA solution in $1 \times$ TBST. Afterwards, the membrane was rinsed and immunoblotted with specific primary antibodies. Antibodies for $\beta$-actin from Cell Signaling Technology (USA), the antibody for LRRC59 was purchased from Abcam (ab127912). And incubations of the appropriate secondary antibody (Cell signaling Technology) for $1 \mathrm{~h}$ at room temperature. Antibodies were used at 1:1000 dilutions according to the manufacturer's instructions. Immunoreactive proteins were detected using an enhanced chemiluminescent (ECL) reagent (Thermo Scientific, Rockford, USA) and images were captured using Azure Biosystem C600 (Azure Biosystems, CA, USA). 


\section{CCK-8 Assay}

Cell viability was assessed by Cell Counting Kit 8(CCK8, Dojindo, Japan). Briefly, cells were seeded in 96-well plate at a density of 1,000 cells/well and incubated at $37{ }^{\circ} \mathrm{C}$ for 24 to $96 \mathrm{~h} ; 10 \mu \mathrm{L}$ CCK-8 solution was added to each well, followed by incubation for $3 \mathrm{~h}$. The absorbance value of each well was measured at $450 \mathrm{~nm}$. Each sample was assayed in four duplicates.

\section{Colony-Formation Assay}

LRRC59 knockdown and control cells were digested by trypsin and seeded in a 6-well plate at a density of 300 cells/well. All the cells were cultured for ten days. Then the clones were washed by PBS, fixed in 4\% paraformaldehyde, stained with $0.1 \%$ crystal violet and counted by Image J. Data represent the average of three independent experiments.

\section{Cell Cycle Analysis}

The analysis of cell cycle was used by Cell Cycle Analysis kit (BD Biosciences, Shanghai, China) and measured according to their protocols. In brief, cells were harvested at $96 \mathrm{~h}$ after infection and resuspended in PBS containing $1 \%$ FBS. Cells $\left(1 \times 10^{6}\right.$ to $\left.3 \times 10^{6}\right)$ were fixed with $70 \%$ ethanol and washed twice before incubating for $15 \mathrm{~min}$ at $37^{\circ} \mathrm{C}$ with propidium iodide (PI)/RNase A solution $(5 \mu \mathrm{g} / \mathrm{mL} \mathrm{PI} / 250 \mu \mathrm{g} / \mathrm{mL}$ RNase $\mathrm{A}$ in $\mathrm{PBS} / 1 \% \mathrm{FBS})$. The incorporation of PI was quantified by FACScan (BD FACS Canto II). The percentage of cells in each phage of the cell cycle was assessed using Modfit LT software.

\section{Apoptosis Analysis}

To detect apoptosis, the expression of Annexin $V /$ propidium Iodide $(P I)$ was evaluated using the Annexin V-FITC Apoptosis Detection kit (BD pharmingen, USA). Briefly, cells were gently detached, then harvested and washed with cold PBS. Cells $\left(7 \times 10^{4}\right)$ were then suspended in $200 \mu \mathrm{L}$ binding buffer containing Annexin V, and incubated for $10 \mathrm{~min}$ at room temperature. After centrifugation (300 g, $3 \mathrm{~min}$ ), cells pellet was resuspended in $200 \mu \mathrm{L}$ binding buffer containing $5 \mu \mathrm{g} / \mathrm{mL}$ propidium iodide, and stained with Annexin V-FITC and PI. Then cells were analyzed using flow cytometry in three different experiments (BD FACS Canto II).

\section{Transwell Assay}

Cell migration ability of A549/H1299 cells was estimated by transwell assay using Transwell chamber with pore size of $8.0 \mu \mathrm{m}$ (Millipore) according to the manufacturer's instructions. Cell invasion ability was performed by using Transwell plates coated with Matrigel (BD Biosciences, USA). $1 \times 10^{5}$ constructed cell clones were suspended in serum-free medium and plated on transwell chambers. The medium containing $10 \%$ FBS was added to the lower chamber as chemoattractant. After $24 \mathrm{~h}$, the chambers were stained with $1 \%$ crystal violet solution for $15 \mathrm{~min}$ and immersed in PBS for $10 \mathrm{~min}$. Then, the cells in the lower chamber were observed and counted under an inverted microscope. The values are expressed as the mean cell numbers under five random fields of view $(200 \times)$. Three independent experiments were conducted for the same conditions.

\section{Statistic Analysis}

All data were analyzed using SPSS pack 26.0 statical software and Graphpad Prism 5.0 software. Survival analyses Bivariate comparisons of clinicopathlogical features between patients with high or low LRRC59 scores were performed using $\chi^{2}$-test. The association of multiple prognostic factors with cancer-specific survival was accessed using univariate and multivariate Cox proportional hazards regression model analysis. Survival analyses were performed using Kaplan-Meier curves and Log rank test. Data are presented as mean \pm standard deviation of at least three independent experiments. Between-groups comparisons were performed using Student's t-tests, $\mathrm{P}<0.05$ was considered to indicate a statistically significant difference.

\section{Results}

\section{High LRRC59 mRNA Level Was} Associated with Worse Prognosis in LUAD To evaluate the relationship between LRRC59 and lung cancer, analysis using the GEPIA2 online tool indicated an upregulated trend of LRRC59 mRNA expression in lung adenocarcinoma and lung squamous cell carcinomas compared with that in normal samples (Figure 1A). Moreover, high mRNA expression of LRRC59 was significantly associated with worse survival in lung cancer patients (Figure 1B). Interestingly, the higher mRNA expression level of LRRC59 was associated only with poor OS for LUAD, but not for lung squamous cell carcinoma (Figure $1 \mathrm{C}$ and $\mathrm{D}$ ). 
A

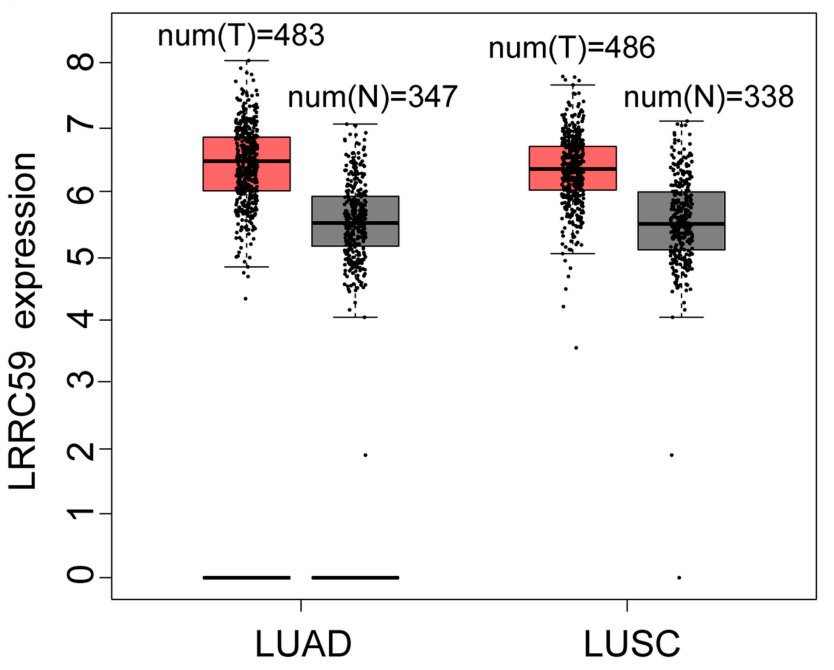

C

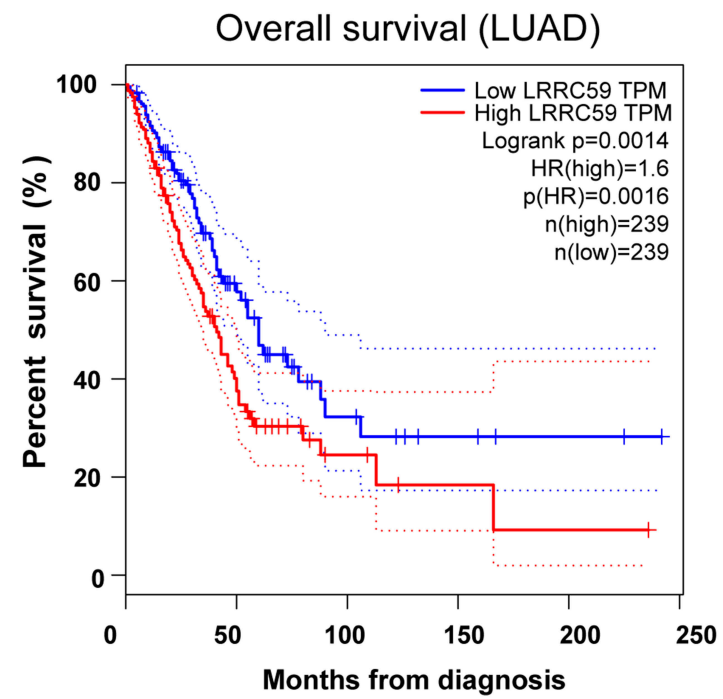

B

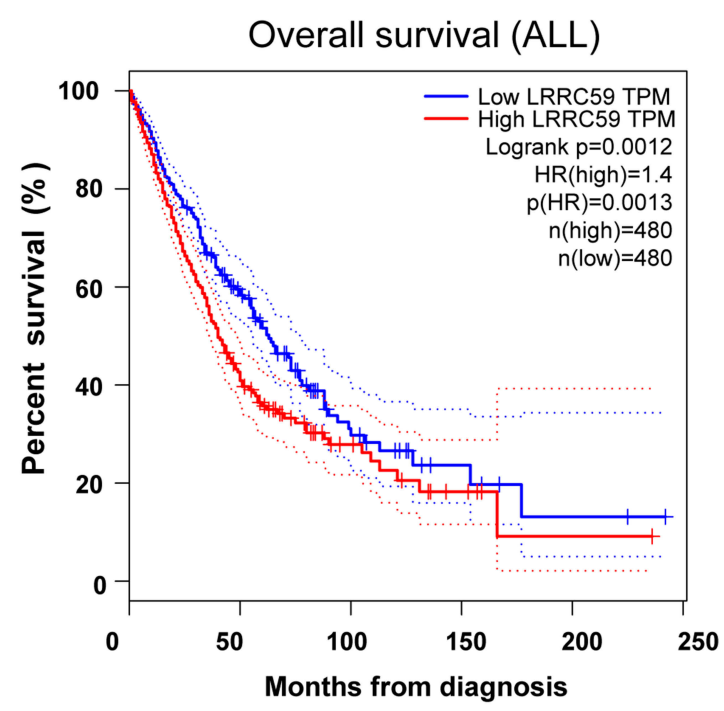

D

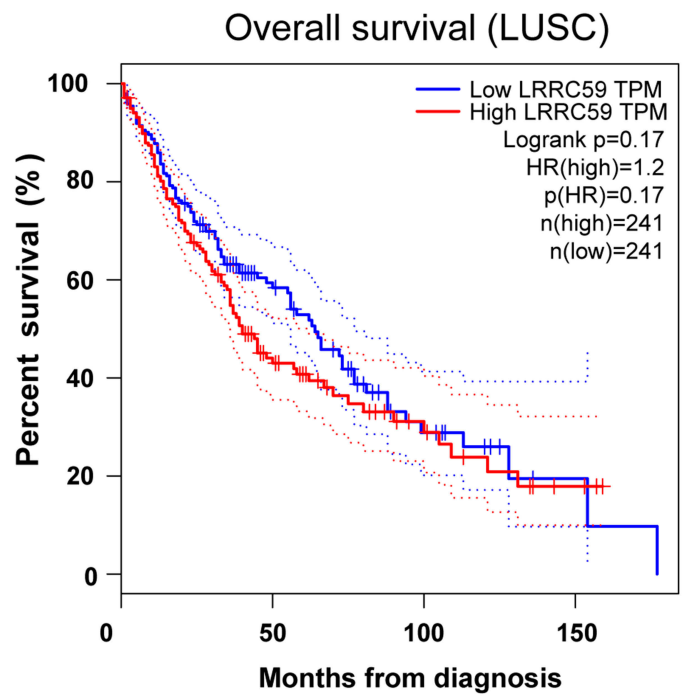

Figure I The prognostic value of the mRNA expression of LRRC59 by online tool GPEIA2 (http://gepia2.cancer-pku.cn). (A) LRRC59 mRNA expression levels in lung adenocarcinoma and lung squamous cell carcinomas are higher than normal tissues. The signature score is calculated by mean value of log2(TPM + I) of LRRC59 in lung cancer and normal tissues. The $\mid$ Log $2 \mathrm{FC} \mid$ cutoff of the expression of proposed biomarker was I. The p-value cutoff of the expression of proposed biomarker was $0.0 \mathrm{I}$. The red box indicates the tumor samples while the gray one represents the normal tissues. (B) Prognostic HRs of LRRC59 in all lung cancer. Survival curves of LRRC59 in lung adenocarcinoma (C) and lung squamous cell carcinoma (D).

Abbreviations: LUAD, lung adenocarcinoma; LUSC, lung squamous cell carcinoma; TPM, transcripts per million; HR, hazard ratio.

\section{Knockdown of LRRC59 Inhibited Lung Cancer Cell Proliferation and Metastasis}

To explore the function of LRRC59 in lung cancer, we generated LRRC59 knockdown cells by transducing LRRC59 shRNAs into H1299 and A549 cells. As shown in Figure 2A and B, shRNA\#1 led to a substantial decrease of LRRC59 expression in $\mathrm{H} 1299$ and A549 cells at the RNA and protein levels, while shRNA\#2 inhibited LRRC59 expression in A549 cells not well as shRNA\#1. Cell proliferation was tested by CCK-8 assay. The results showed that the proliferation of H1299 and A549 cells in the shLRRC59 group was significantly impaired compared with that in the control group ( $<<0.001$, Figure $2 \mathrm{C}$ and $\mathrm{D}$ ). Colony-formation assays revealed that silencing LRRC59 (shLRRC59) dramatically inhibited the size and number of colonies in both cell lines, compared with the cells in the shCon group (Figure 2E and F).

As proliferation-suppression phenotypes were observed after depletion of LRRC59 by RNA interference, cell cycle distribution was further detected by flow cytometry. Consistently, A significant increase in the G1 phase was observed in A549 and H1299 cells transduced with shLRRC59s compared with control cells (Figure $2 \mathrm{G}$ and $\mathrm{H}$ ). 
A

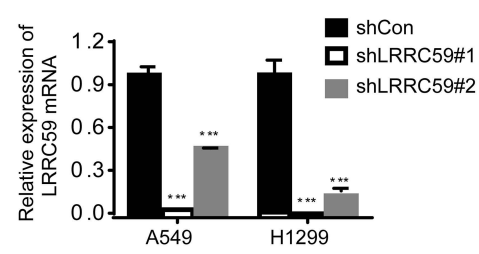

C

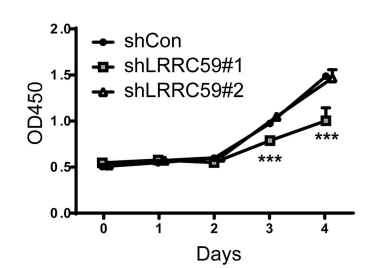

E
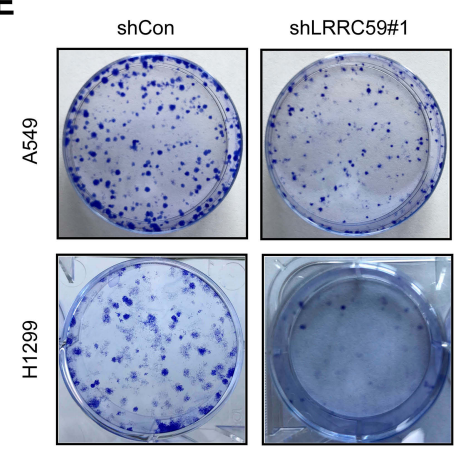

G
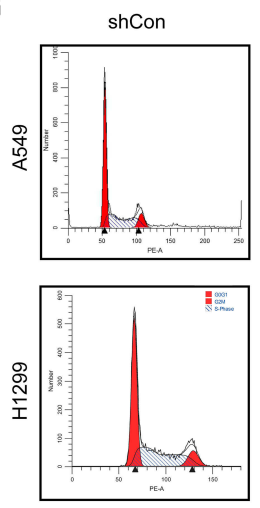

ShLRRC59\#1
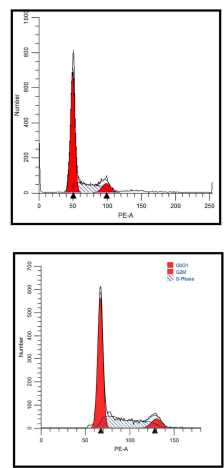

shLRRC59\#2
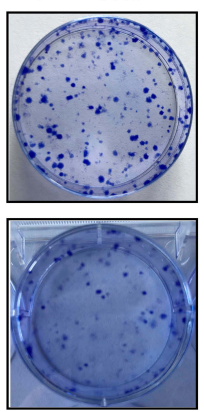

ShLRRC59\#2
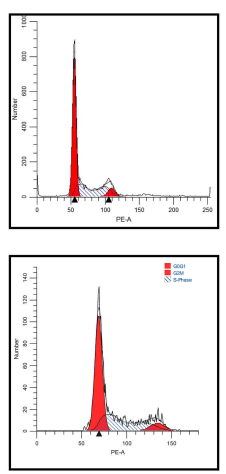

B

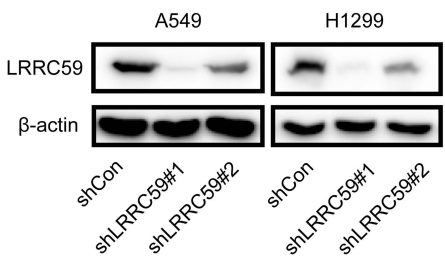

H1299

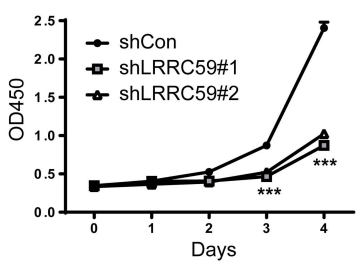

F
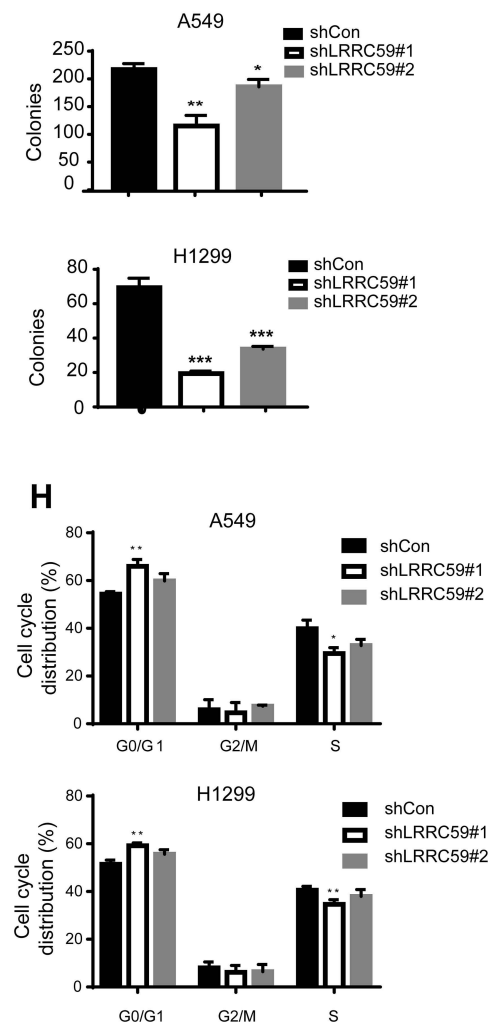

Figure 2 Knockdown of LRRC59 inhibited the proliferation and induced GI/S phase arrest (A) qRT-PCR results of LRRC59 expression in shRNA-transduced cells. LRRC59 expression was normalized to $\beta$-actin. After 72-hour post-transduction with shRNA, the cells were subjected to qRT-PCR. The data were calculated from three independent experiments $(* * * \mathrm{P}<0.00 \mathrm{I})$. (B) Western-blot analysis of LRRC59 protein expression in lung cancer cells after LRRC59 knockdown. $\beta$-actin was used as a control. (C, D) Cell viability of lung cancer cells following LRRC59 knockdown and corresponding control cells. After transduction with shRNAs, cells were seeded into 96 -well plates at 2000 cells/well and determined for the indicated times. The data were calculated in triplicate vs control (***P<0.00I). (E, F) Representative results of colony-formation assays in lung cancer cells. The numbers of colonies are shown in the right panel (F). After 72-hour transduction with shRNAs, cells were seeded into six-well plates at I 000 cells/well and cultured for ten days. Stained colonies with more than 50 cells were considered colony-forming units. The experiment was performed in triplicate wells in three independent experiments $(* \mathrm{P}<0.05$; $* * \mathrm{P}<0.0 \mathrm{I} ; * * * \mathrm{P}<0.00 \mathrm{I})$. (G, $\mathbf{H})$ Flow cytometry analysis of cell cycle in shCon and shLRRC59 lung cancer cells. After 72-hour post-transduction with shRNA, the cells were subjected to cell cycle analysis. Data were representative results from experiments repeated at least three times $(* \mathrm{P}<0.05$; $* * \mathrm{P}<0.01$ vs shCon group).

Abbreviations: sh, short hairpin RNA; shCon, shRNA-control; OD, optical density; PE-A, phycoerythrin-A; GI/S, gap phasel/synthesis phase; G0/GI, gap phase0/gap phasel; G2/M, gap phase2/mitosis phase.

Furthermore, the effects of LRRC59 on the migrated and invasive ability of lung cancer cells were assessed by Transwell assay. As shown in Figure 3, the silencing of LRRC59 in A549 and H1299 cells caused a significant reduction in cell migration (Figure 3A and $\mathrm{B}$ ) and cell invasion (Figure $3 \mathrm{C}$ and D). These data indicated that LRRC59 plays a vital role in cancer cell migration, invasion, and tumor progression. 
A

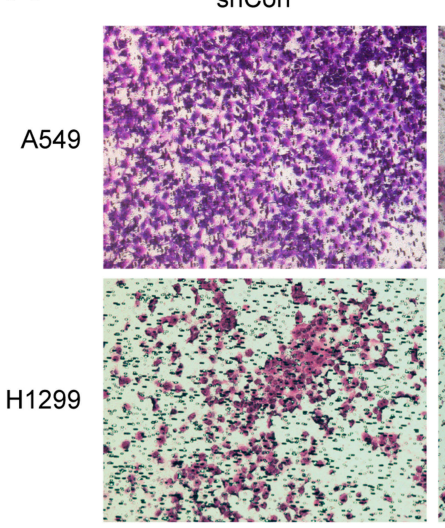

C

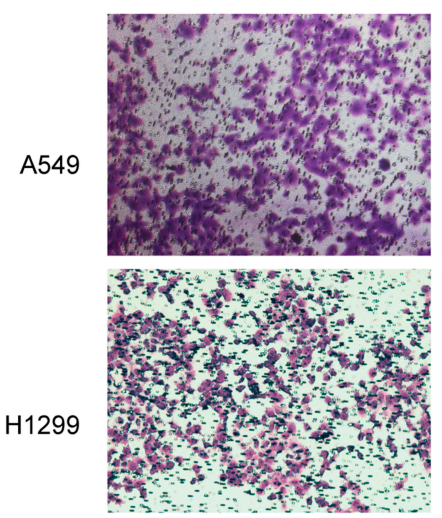

shLRRC59\#1
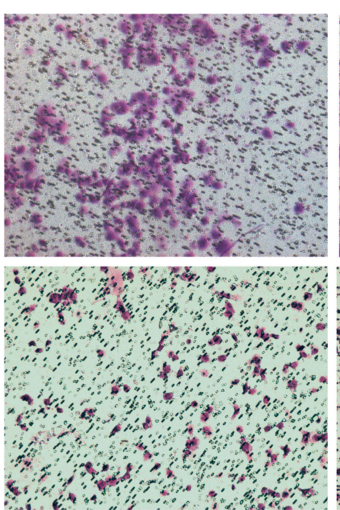

shLRRC59\#1

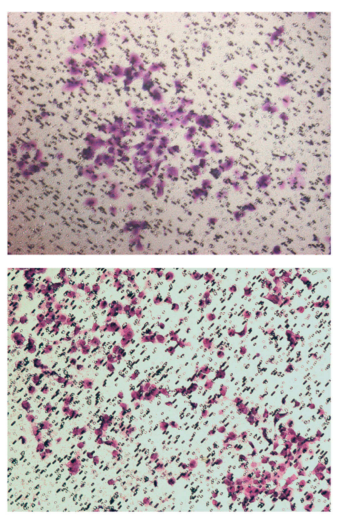

ShLRRC59\#2

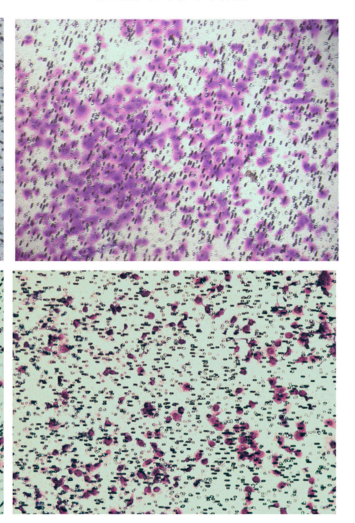

ShLRRC59\#2

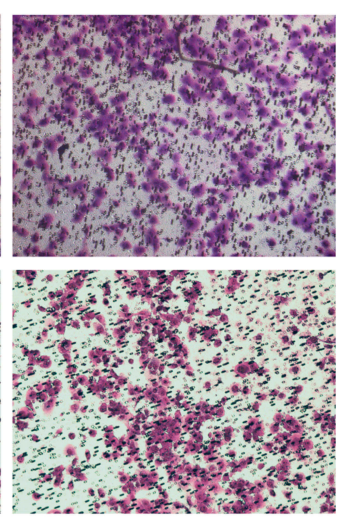

B
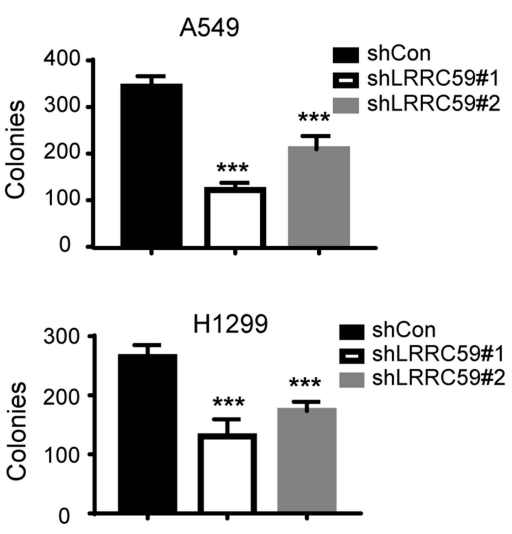

D
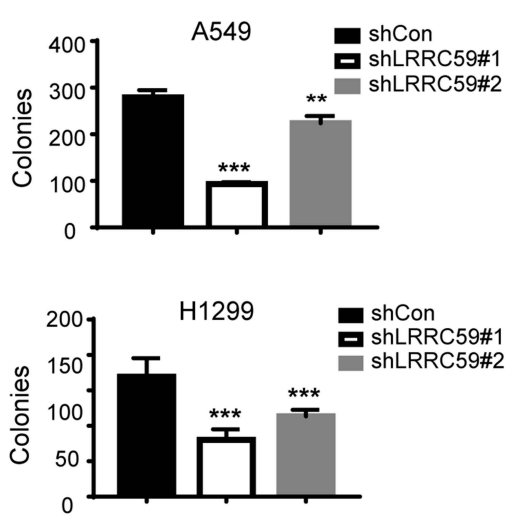

Figure 3 Knockdown LRRC59 impaired the migratory and invasive abilities of lung cancer cells. (A, B) Transwell migration analysis of shCon and shLRRC59 lung cancer cells. Representative images of migration assays in A549 and HI299 cells (magnification, 200x) are shown. And histograms of migration results were shown in (B). Data were representative results from experiments repeated at least three times $(* * * \mathrm{P}<0.00 \mathrm{I})$. (C, D) Transwell invasion analysis of shCon and shLRRC59 lung cancer cells. Data were representative results from experiments repeated at least three times (**P $<0.0 \mathrm{I}$; $* * * \mathrm{P}<0.00 \mathrm{I})$.

Abbreviations: shCon, short hairpin RNA-control; sh, short hairpin RNA.

\section{LRRC59 Depletion Induced the Apoptosis of A549 Cells, but Not P53-Deficient HI299 Cells}

To estimate the effect of LRRC59 knockdown on apoptosis, Annexin V-FITC/PI double staining and flow cytometry was performed in A549 and H1299 cells (Figure 4). Our result showed that the apoptosis rate of LRRC59 knockdown in A549 cells was more significantly higher than that in control cells, while silencing LRRC59 expression has a slight effect on apoptosis in H1299 cells.

\section{LRRC59 Serves as an Independent Factor for the Prediction of Worse Survival in LUAD}

Our results revealed that LRRC59 is an oncogenic factor that contributes to cancer proliferation and metastasis. The online survival tool analysis (GEPIA2) also indicated that LUAD patients with high mRNA levels of LRRC59 had poorer prognosis. To further confirm that LRRC59 could be an independent risk factor of worse outcomes in LUAD patients, the expression and localization of LRRC59 were analyzed by TMA-based IHC. The expression of LRRC59 was mainly localized in the cancer cell cytoplasm (Figure 5A-C); Whereas, LRRC59 expression in the nuclei was sparse. Additionally, we compared 94 lung adenocarcinoma specimens with paired adjacent normal tissue in a large microarray, the average staining score of tumor tissue (avg $=5.47$ ) was significantly higher than adjacent tissue (avg $=1.48, \mathrm{P}<0.001$, Figure 5D).

Then, the association between LRRC59 expression and the clinicopathological variables of 94 patients was analyzed. The level of LRRC59 expression was divided into low group $(n=38)$ and high group $(n=56)$ according to the cutoff value stated in methods mentioned before. The data 

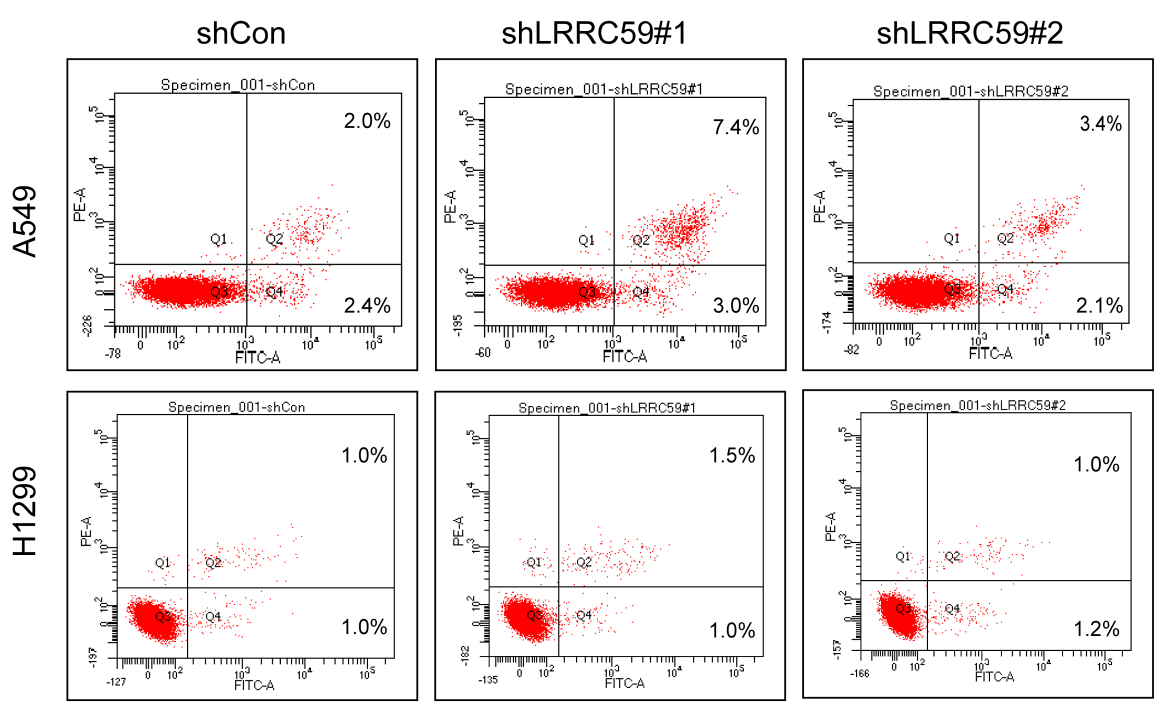

Figure 4 Knockdown LRRC59 induced the apoptosis of A549 cells, but not p53-deficient HI299 cells. Apoptosis in shCon and shLRRC59 lung cancer cells were collected for Annexin $\mathrm{V}$ and $\mathrm{Pl}$ staining, followed by flow cytometry analysis.

Abbreviations: PE-A, phycoerythrin-A; FITC-A, fluorescein isothiocyanate-A; PI, propidium iodide; shCON, short hairpin RNA-control; sh, short hairpin RNA.

are summarized in Table 1. High LRRC59 expression was closely associated with lymph node metastasis $(\mathrm{P}<0.001)$, TNM stage $(\mathrm{P}<0.001)$, histological differentiation $(\mathrm{P}=0.007)$, while there was no correlation with age $(\mathrm{P}=0.078)$, gender $(\mathrm{P}=0.495)$, tumor size $(\mathrm{P}=0.147)$.

Furthermore, we employed Kaplan-Meier analysis to evaluate the relationship between LRRC59 expression and OS in LUAD patients. The results showed that patients with high LRRC59 expression displayed a shorter overall survival than those of low LRRC59 expression $(\mathrm{P}=0.02)$ (Figure 5E), which was similar to that of the GEPIA2 online prediction. Importantly, multivariate Cox regression analysis indicated that high expression of LRRC59 protein was an independent prognostic factor of LUAD (Table 2).

\section{Discussion}

LUAD is one of the most aggressive and rapidly mortal tumor types with overall survival less than five years. The poor prognosis of LUAD is mainly due to late diagnosis and indistinct mechanism of cancer development. The identification of novel therapeutic targets in LUAD is urgently needed.

Recently, Yu et al showed that a fusion transcript of LRRC59-FLJ60017 among seven other fusion transcripts is strongly associated with prostate cancer prognosis A combination of these fusion transcripts in prostate cancer with Gleason's grading was predictive of prostate carcinoma recurrence. In this study, we found that LRRC59 is widely upregulated in LUAD specimens and correlates with TNM stage, lymph node metastasis, histological differentiation, and independently predicts shorter overall survival in LUAD patients. Combined with the predictive results of biological information, we speculate that LRRC59 might play a tumor-promoting role in the LUAD process.

Although the altered expression of LRRC59 proteins has been reported in a variety of human cancers, ${ }^{8,9}$ the primary function of LRRC59 is largely unknown at present. LRRC59 was initially identified as an intracellular binding partner of Fibroblast Growth Factor 1 (FGF1) and required for the nuclear import of FGF $1 .^{5}$ It is well known that FGF1 is wildly involved in cell proliferation, survival, migration and invasion, ${ }^{10}$ which is easily hijacked and rewired by cancer cells to foster tumor growth, survival, and metastasis. Our data showed that knockdown of LRRC59 significantly inhibited the proliferation and induced G1/S phase arrest of LUAD cells. Moreover, we found that silencing LRRC59 induced the apoptosis of A549 cells, but not p53-deficient H1299 cells. Consistently, previous reports have shown that intracellular FGF1 inhibits both p53-dependent apoptosis and cell growth arrest via an intracrine pathway. ${ }^{10-13}$ However, we did not detailed the FGF1 pathways in our study, whether LRRC59 modulates LUAD progression via FGF1 pathway remains further exploration.

Previous studies have shown that the upregulation of LRRC59 was correlated with the metastatic capability of breast cancer cell lines, ${ }^{7,8,14}$ the silencing of LRRC59 caused an evident transition from a mesenchymal phenotype toward 

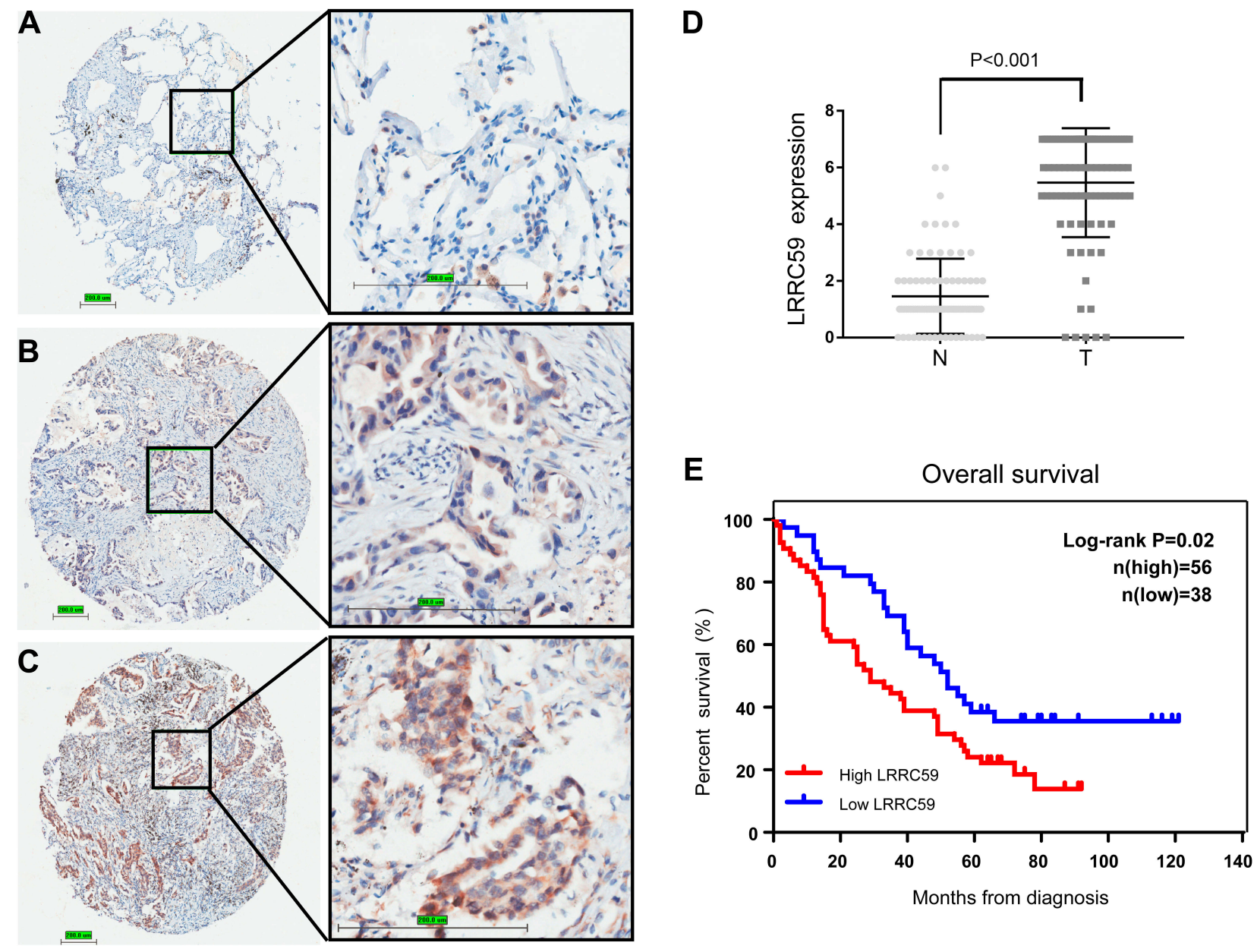

Figure 5 High LRRC59 expression is associated with worse prognosis in lung adenocarcinoma patients. (A-C) Representative microphotographs of LRRC59 immunohistochemical staining in lung tissues negative stain of LRRC59 (A), lung adenocarcinomas were weakly positive for LRRC59 (B) and strongly positive staining of LRRC59 (C). (D) LRRC59 expression scores in LUAD tissues and adjacent tissues. LRRC59 expression score is the sum of the staining intensity and positive percentage (N, adjacent tissues; T, lung tumor tissues). (E) Kaplan-Meier curves of overall survival for LUAD patients with high LRRC59 expression or low LRRC59 expression. Kaplan-Meier analysis for overall survival was performed with P-values determined by Cox proportional hazards regression.

Abbreviation: LUAD, lung adenocarcinoma.

an epithelial one, and strongly impaired wound closure. Consistently, we found that LRRC59 is widely upregulated in LUAD specimens and positively correlates with lymph node metastasis. Moreover, a significant decrease in migration and invasion were observed in both A549 and H1299 cells with LRRC59 knockdown. Thus, we preliminarily

Table 2 Univariate and Multivariate Cox Proportional Hazards Analysis of LRRC59 Expression and OS for LUAD Patients

\begin{tabular}{|l|l|l|l|l|l|l|}
\hline \multirow{2}{*}{ Variable } & \multicolumn{2}{l|}{ Univariate Analysis } & \multicolumn{2}{l|}{ Multivariate Analysis } \\
\cline { 2 - 7 } & Hazard Ratio & $\mathbf{9 5 \%}$ Confidence Interval & P value & Hazard Ratio & 95\% Confidence Interval & P value \\
\hline Age & 0.950 & $0.594-1.520$ & 0.832 & & & \\
Gender & 1.364 & $0.850-2.189$ & 0.198 & & \\
Tumor size & 1.357 & $0.839-2.196$ & 0.214 & & & \\
Differentiation & 1.626 & $1.038-2.545$ & $0.034^{\mathrm{a}}$ & 1.175 & $0.708-1.948$ & \\
LN metastasis & 3.206 & $1.898-5.416$ & $<0.001^{\mathrm{a}}$ & 2.516 & $1.457-4.344$ & 0.533 \\
LRRC59 & 2.786 & $1.648-4.712$ & $<0.001^{\mathrm{a}}$ & 2.009 & $1.144-3.526$ & $0.00 \mathrm{I}^{\mathrm{a}}$ \\
\hline
\end{tabular}

Notes: Statistical were performed by Cox regression analysis. ${ }^{\mathrm{a}} \mathrm{P}<0.05$ was considered significant. 
determined that LRRC59 is a poor prognostic factor for LUAD, and might serve a key role in LUAD metastasis.

Recently, LRRC59 is attracting more attention as a potential oncogene involved in nuclear import of FGF1 and the cancerous inhibitor of PP2A (CIP2A) ${ }^{6}$. FGF1 and CIP2A is over-expressed in a variety of cancers, ${ }^{15-18}$ including lung cancer. In addition, LRRC59 gene amplification or protein overexpression is observed in several kinds of cancers. ${ }^{7-9,14}$ In this study, we showed that LRRC59 is widely upregulated in LUAD specimens and associated with poor prognosis. This is the first research on LRRC59 effects on prognosis of LUAD. We hope this study can trigger more interest in LRRC59 function and targeted therapy in LUAD. Unfortunately, this research focused on clinical study of the correlation between LRRC59 and LUAD prognosis, and did not determine whether LRRC59 modulates LUAD progression via FGF1 or CIP2A pathway. More experiments are needed to elucidate downstream signaling pathways initiated by LRRC59 as well as the entire signaling network of LRRC59. In addition, the animal model is an essential tool for studying the role of biomarkers in lung cancer progression. We hope that our results in vitro can trigger further investigation of the role of LRRC59 in LUAD in vivo.

\section{Conclusion}

In conclusion, we initially reported that LRRC59 was overexpressed in LUAD tissues compared with adjacent nontumorous lung tissues, and it was significantly associated with TNM stage, lymph node metastasis, histological differentiation and unfavorable prognosis of LUAD. In addition, knockdown of LRRC59 could substantially inhibit lung cancer cell proliferation, colony formation, migration, and invasion by in vitro study. Together, our findings indicate that LRRC59 may represent a potential biomarker and a promising therapeutic target for LUAD.

\section{Acknowledgments}

This work was supported by grants from the Technology Project of Medical \& Health Science in Zhejiang province (2019RC286).

\section{Disclosure}

The authors report no conflicts of interest in this work.

\section{References}

1. Jemal A, Siegel R, Xu J, et al. Cancer statistics. CA Cancer J Clin. 2010;60(5):277-300. doi:10.3322/caac.20073

2. Skjerpen CS, Wesche J, Olsnes S. Identification of ribosome-binding protein p34 as an intracellular protein that binds acidic fibroblast growth factor. J Biol Chem. 2002;277(26):23864-23871. doi:10.10 74/jbc.M112193200

3. Ichimura $\mathrm{T}$, Ohsumi $\mathrm{T}$, Shindo $\mathrm{Y}$, et al. Isolation and some properties of a 34-kDa-membrane protein that may be responsible for ribosome binding in rat liver rough microsomes. FEBS Lett. 1992;296(1):7-10.

4. Ohsumi T, Ichimura T, Sugano $\mathrm{H}$, et al. Ribosome-binding protein p34 is a member of the leucine-rich-repeat-protein superfamily. Biochem J. 1993;294(2):465.

5. Zhen Y, Sorensen V, Skjerpen CS, et al. Nuclear import of exogenous FGF1 requires the ER-protein LRRC59 and the importins Kpnalpha1 and Kpnbeta1. Traffic. 2012;13(5):650-664. doi:10.1111/j.16000854.2012.01341.x

6. Pallai R, Bhaskar A, Barnett-Bernodat N, et al. Leucine-rich repeat-containing protein 59 mediates nuclear import of cancerous inhibitor of PP2A in prostate cancer cells. Tumour Biol. 2015;36 (8):6383-6390. doi:10.1007/s13277-015-3326-1

7. Yu YP, Liu P, Nelson J, et al. Identification of recurrent fusion genes across multiple cancer types. Sci Rep. 2019;9:9.

8. Terp MG, Lund RR, Jensen ON, et al. Identification of markers associated with highly aggressive metastatic phenotypes using quantitative comparative proteomics. Cancer Genomics Proteomics. 2012;9(5):265-273.

9. Zefang T, Boxi K, Chenwei L, et al. GEPIA2: an enhanced web server for large-scale expression profiling and interactive analysis. Nucleic Acids Res. 2019;47(W1):556-560. doi:10.1093/nar/gkz430

10. Turner N, Grose R. Fibroblast growth factor signalling: from development to cancer. Nat Rev Cancer. 2010;10(2):116-129. doi:10.1038/ nrc2780

11. Yan PY, Ding Y, Chen Z. Novel fusion transcripts associate with progressive prostate cancer. Am J Pathol. 2014;184(10):2840-2849. doi:10.1016/j.ajpath.2014.06.025

12. Bouleau S, Grimal H, Rincheval V, et al. FGF1 inhibits p53-dependent apoptosis and cell cycle arrest via an intracrine pathway. Oncogene. 2005;24(53):7839-7849. doi:10.1038/sj.onc. 1208932

13. Pirou C, Montazer-Torbati F, Jah N, et al. FGF1 protects neuroblastoma SH-SY5Y cells from p53-dependent apoptosis through an intracrine pathway regulated by FGF1 phosphorylation. Cell Death Dis. 2017;8(8):e3023. doi:10.1038/cddis.2017.404

14. Rodriguez-Enfedaque A, Bouleau S, Laurent M, et al. FGF1 nuclear translocation is required for both its neurotrophic activity and its p53-dependent apoptosis protection. Biochim Biophys Acta Bioenerg. 2009;1793(11):1719-1727. doi:10.1016/j.bbamcr.2009.09.010

15. Li J, Wei Z, Li H, et al. Clinicopathological significance of fibroblast growth factor 1 in non-small cell lung cancer. Hum Pathol. 2015;46 (12):1821-1828. doi:10.1016/j.humpath.2015.07.022

16. Vaarala MH, Väisänen M-R, Ristimäki A. CIP2A expression is increased in prostate cancer. J Exp Clin Cancer Res. 2010;29 (1):136. doi:10.1186/1756-9966-29-136

17. Böckelman C, Lassus H, Hemmes A, et al. Prognostic role of CIP2A expression in serous ovarian cancer. Br J Cancer. 2011;105 (7):989-995. doi:10.1038/bjc.2011.346

18. Teng HW, Yang SH, Lin JK, et al. CIP2A is a predictor of poor prognosis in colon cancer. $J$ Gastrointest Surg. 2012;16 (5):1037-1047. doi:10.1007/s11605-012-1828-3 


\section{Publish your work in this journal}

OncoTargets and Therapy is an international, peer-reviewed, open access journal focusing on the pathological basis of all cancers, potential targets for therapy and treatment protocols employed to improve the management of cancer patients. The journal also focuses on the impact of management programs and new therapeutic

Submit your manuscript here: https://www.dovepress.com/oncotargets-and-therapy-journal agents and protocols on patient perspectives such as quality of life, adherence and satisfaction. The manuscript management system is completely online and includes a very quick and fair peer-review system, which is all easy to use. Visit http://www.dovepress.com/ testimonials.php to read real quotes from published authors. 